\title{
Heterosis and Combining Ability Analysis Oil Content Seed Yield and its Component in Linseed
}

\author{
Shalendra Kumar*, P.K. Singh, S.D. Dubey, S.K. Singh and Alankar Lamba \\ Department of Genetics and Plant Breeding, Chandra Shekhar Azad University of \\ Agriculture and Technology, Kanpur-208 002, India \\ *Corresponding author
}

\section{A B S T R A C T}

This study was undertaken to estimate the combining ability in linseed through diallel analysis involving eight diverse genotypes. A $8 \times 8$ full diallel crosses study, including the reciprocals, with linseed (Linum usitatissimum $\mathrm{L}$.) was performed to determine both the

\section{Keywords}

Dialle, Combining ability, Heterosis and Linum usitatissimum L.

Article Info

Accepted:

12 September 2017

Available Online:

10 November 2017 magnitude of gene action and heterotic performance of the crosses for seed yield, oil content and important yield components. Field experiments were conducted at the investigation research farm, Nawabganj, C. S. Azad University of agriculture and technology Kanpur. All 56 F1 and F2 hybrids and their parents were sown in a randomized complete block design with 3 replicates. Additive genetic variance is the result of additive gene action whereas non additive variance is made up of dominance and epistasis gene action. The mean squares of the general combining ability (GCA), specific combining ability (SCA) and reciprocal combining ability (RCA) were statistically significant for all traits evaluated. The parents RKY-19, OLC-60, PADMINI, TL-27, SJKO-60, T L-11, S36 and KL-231were good general combiner for almost the characteristics evaluated. The significant positive batter-parent heterosis values were obtained with several crosses in important yield components. In conclusion, the parents used in this study exhibited positive GCA effects for seed yield. Therefore they could be considered as promising parents in the production of F1 hybrids and in further breeding studies.

\section{Introduction}

Linseed (Linum usitatissimum L.) is a diploid $(2 \mathrm{n}=30$, genome size $\sim 370 \mathrm{Mb}$ ) selfpollinated annual oilseed plant. It has been under the cultivation for its seed or stem fibre (Flax) of both (dual purpose) for 1000 years (Dillman, 1953). Every part of the linseed plant is utilized commercially either directly or after processing. On a very small scale, the seed is directly used for edible purposes and about $20 \%$ of the total oil produced is used in farmer home. About $80 \%$ of the oil goes to the industries for the manufacturing of rapidly drying paints, varnish, oil cloths, polymer linoleum, pad-ink, printing ink, etc. The oil cake is a good feed for milch cattle. The oil contains different fatty acids like alpha linolenic acid (omega-3) 53.21\%, linoleic acid (omega-6) $17 \%$, oleic acid $18.51 \%$, stearic acid $4.42 \%$ and palmitic/palmitoleic acid 4$6 \%$. Linseed is the richest source of omega-3 fatty acid and it contains almost twice as much as of omega-3 in fish oil. The ratio of omega-3: omega- 6 present in linseed is about $4: 1$, so this is a best herbal source of omega-3 
for improvement in human metabolism (Viorica-MirelaPopa, 2012). Through diallel analysis a number of parental lines can be tested in all possible combinations. Thus, the main objective of the present study was to identify the best combiners and their crosses on the basis of their general and specific combining ability for oil content and its quality parameters. Hybrid is an alternative approach to increase the productivity and most important step in the hybrid breeding program is the detection of suitable parents with high general ( $g c a)$ and specific combining ability $(s c a)$ for grain yield and then the exploitation of heterosis. The study of heterosis has a direct bearing on the breeding methodology to be employed for varietal improvement and also provides useful information about usefulness of the parents in breeding programs.

\section{Materials and Methods}

\section{Experimental material and design}

The material for the investigation comprised of eight improved strains/varieties of linseed namely RKY-19, OLC-60, PADMINI, TL-27, SJKO-60, T L-11, S- 36, KL-231 having desire genetic variability for oil content, yield and associated attribute. Parental seed were collected from Project Coordinating Unit (Linseed) C. S. Azad University. All possible crosses were made during rabi 2012-13 in a complete diallel fashion $(8 \times 8)$. The F1 and F2 along with their parents were grown in randomization block design using three replication during rabi season 2014-15 at the investigation research farm, Nawabganj, C. S. azad University of agriculture and technology Kanpur.

\section{Analysis of variance}

The analysis of variance for the experimental design was based on the model
$\mathrm{P}_{\mathrm{ijk}}=\mu+\mathrm{v}_{\mathrm{ij}}+\mathrm{b}_{\mathrm{k}}=\mathrm{e}_{\mathrm{ijk}}$

$(\mathrm{i}, \mathrm{j}=1 \ldots . ., \mathrm{t} ; \mathrm{k}=1 \ldots . . \mathrm{b})$

Where

$P_{\mathrm{ijk}}=$ the phenotype of $\mathrm{ijk} \mathrm{k}^{\text {th }}$ observation

$\mu=$ the population mean

$\mathrm{v}_{\mathrm{ij}}=$ the progeny effect

$b_{k}=$ the block effect

$\mathrm{e}_{\mathrm{ijk}}=$ the error term for $\mathrm{ijk} \mathrm{k}^{\text {th }}$ observation

On the basis of above model, the data obtained were first subjected to randomized block analysis. The skeleton of analysis of variance is given as under

\section{Combining ability analysis}

Combining ability analysis was performed according to the procedure suggested by Griffing (1956b) Method 1, Model I. In this model parents, direct crosses and reciprocals crosses are included for the analysis.

This method permits estimation of reciprocal differences. It is also assumed that error is independently and normally distributed with the mean zero and error variance $\sigma^{2}$ e. The analysis of variance for combining ability was based on the following mathematical model:

$$
\begin{aligned}
& X_{i j k}=\mu+\hat{g}_{i}+\hat{g}_{j}+\hat{s}_{i j}+b_{k}+e_{i j k} \\
& (\mathrm{i}, \mathrm{j}=1,2 \ldots . ., \mathrm{n} ; 1=1,2, \ldots \ldots \mathrm{b})
\end{aligned}
$$

Where

$\mu=$ the population mean

$\hat{\mathrm{g}}_{\mathrm{i}}=$ the general combining ability (gca) for $\mathrm{i}^{\text {th }}$ parent

$\hat{\mathrm{g}}_{j}=$ the gca of the $\mathrm{j}^{\text {th }}$ parent 
$\hat{s}_{i j}=$ the specific combining ability (sca) for the cross between the $\mathrm{i}^{\text {th }}$ and $\mathrm{j}^{\text {th }}$ parents such that $\mathrm{s}_{\mathrm{ij}}=\mathrm{s}_{\mathrm{ji}}$

$b_{k}=$ block effect

$\mathrm{e}_{\mathrm{ijk}}=$ the environmental effect associated with the $\mathrm{ijk}^{\text {th }}$ individual observation on $\mathrm{i}^{\text {th }}$ individual in $\mathrm{k}^{\text {th }}$ block with $\mathrm{i}^{\text {th }}$ as female parent and $\mathrm{j}^{\text {th }}$ as male parent.

$\mathrm{b}=$ number of blocks/replications

The restrictions imposed on this model are:

$\sum_{i} g_{i}=0$ and $\sum_{j} g_{i j}+s_{i i}=0$

(For each $\mathrm{i}$ ), where $\mathrm{i}=$ variety

Where,

$\mathrm{b}=$ number of replications

$\mathrm{c}=$ number of progenies (parents $+\mathrm{F}_{1} \mathrm{~s}$ )

$\mathrm{r}=$ number of reciprocals

$S_{g}=\frac{1}{n+2}\left[\sum_{i}\left(x_{i}+x_{i i i}\right)^{2}+\frac{2}{(n+1(n+2)} x^{2}\right] .$.

$\mathrm{M}^{\prime} \mathrm{e}=\mathrm{Me} / \mathrm{bc}$

Where,

$\mathrm{b}=$ number of replications

$\mathrm{c}=$ number of observations per plot

$\mathrm{M}_{\mathrm{e}}=$ the error m.s.s. obtained from previous ANOVA

$\mathrm{S}_{\mathrm{g}}=$ the sum of squares (s.s.) due to gca

$\mathrm{S}_{\mathrm{s}}=$ the sum of squares (s.s) due to sca

$\mathrm{n}=$ numbero $\mathrm{f}$ parents

$\mathrm{x}_{\mathrm{i} .}=$ total of array involving $\mathrm{i}^{\text {th }}$ as female $\mathrm{x}_{\mathrm{ii}}=$ the value of the $\mathrm{i}^{\text {th }}$ parent of the array

$\mathrm{x} . .=$ the grand total

$\mathrm{x}_{\mathrm{ij}}=$ the value of the cross with $\mathrm{i}^{\text {th }}$ as female and $\mathrm{j}^{\text {th }}$ as male parents.

Estimates of various effects

General Combining Ability Effects (GCA)

gi $=(1 / 2)(X i .+X . i)-X . . / n^{2}$

Where:

$\mathrm{g}_{\mathrm{i}}=$ General combining ability effects for line F1's i.

$\mathrm{n}=$ Number of parents/varieties

$\mathrm{X}_{\mathrm{i}} .=$ Total of mean values of $\mathrm{F}_{1}$ 's resulting from crossing $j$ th lines with $i$ th lines.

$\mathrm{X}_{\cdot \mathrm{i}}=$ Total of mean values of $\mathrm{F}_{1}$ 's resulting from crossing the $i$ th line with the $j$ th line.

$\mathrm{X}=$ Grand mean of all the mean values in the table

Specific Combining Ability Effects (SCA)

$s_{\mathrm{ij}}=(1 / 2)\left(X_{\mathrm{ij}}+\mathrm{X}_{\mathrm{ji}}\right)-(1 / 2)\left(\mathrm{X}_{\mathrm{i} .}+\mathrm{X}_{. \mathrm{i}}+\mathrm{X}_{\mathrm{j} .}+\mathrm{X}_{. \mathrm{j}}\right)+$ $\mathrm{X} . . / \mathrm{n} 2$

Where:

$s_{i j}=$ Specific combining ability between $i$ th and $j$ th lines.

$X_{i j}=$ Mean value of the $F_{1}$ resulting from crossing the $i$ th and $j$ th lines.

$X_{\mathrm{ji}}=$ Mean values for $F_{1}$ resulting from crossing the $j$ th and $i$ th lines. 
$X_{i .}=$ Total of means of $F_{1}$ 's resulting from crossing $j$ th line with $i$ th line.

$\mathrm{X}_{\cdot \mathrm{i}}=$ Reciprocal values of $\mathrm{Y}_{\mathrm{i}}$.

$X_{j}$. = Total valves for $F_{1}$ 's resulting from crossing the $i$ th line with $j$ th line.

$X_{. j}=$ Values of reciprocal $F_{1}$ 's of $Y_{. j}$.

X. $=$ Grand values of the observations

\section{Reciprocal Effects (REC)}

$\mathrm{r}_{\mathrm{ij}}=\left(\mathrm{X}_{\mathrm{ij}}-\mathrm{X}_{\mathrm{ji}}\right) / 2$

Where:

$\mathrm{r}_{\mathrm{ij}}=$ Reciprocal effects of the $i$ th and $j$ th lines.

$X_{i j}=$ Mean values for the $F_{1}$ resulting from crossing the $i$ th and $j$ th lines.

$\mathrm{X}_{\mathrm{ji}}=$ Reciprocal effects of $\mathrm{F}_{1}$ resulting from $\mathrm{Xij}$.

Estimated variances of the estimates of the effect and their differences:

Esti. Var. $\left(\hat{g}_{i}\right)=\frac{n-1}{2 n 2} \hat{\sigma}_{e}^{2}$

Esti. Var. $\left(\hat{s}_{\mathrm{ij}}\right)=\frac{\left(\mathrm{n}^{2}+\mathrm{n}+2\right)}{(\mathrm{n}+1)(\mathrm{n}+2)} \hat{\sigma}_{\mathrm{e}}^{2}$, where $\quad \mathrm{i} \neq \mathrm{j}$

Esti. Var. $\left(\hat{g}_{\mathrm{i}} \hat{\mathrm{g}}_{\mathrm{j}}\right)=\frac{2}{\mathrm{n}+2} \hat{\mathrm{\sigma}}_{\mathrm{e}}^{2}$, where $\quad \mathrm{i} \neq \mathrm{j}$

Esti. Var. $\left(\hat{s}_{i j}-\hat{s}_{i k}\right)=\frac{2 n}{n+2} \hat{\sigma}_{e}^{2}$, where $\quad i \neq j$

\section{Estimation of heterosis}

The magnitude of heterosis was calculated with the help of the formulae given below:
Heterosis over better parent $(\%)=$ $\frac{\overline{F_{1}}-\bar{B} \bar{P}}{\bar{B} \bar{P}} \times 100$

Where,

$\mathrm{BP}=$ the value of the better parent.

\section{Analysis of variance}

The analysis of variance for combining ability (Table 1) revealed highly significant variance for both general and specific combining ability in both generations for all the characters, indicating the importance of both additive and non-additive gene action in the expression of these traits. Reciprocal effects of maternal and paternal combining ability showed that use in both form of parent for almost characters. However, additive and non-additive effects were predominant for all the characters, as reported by various workers Singh et al., (2008), Brahm Singh et al., (2008), Singh et al., (2009), Pali and Mehta (2014),

Additive genetic variance is the result of additive gene action whereas non additive variance is made up of dominance and epistasis gene action. The dominance variance decline by half with each other generation of selfing or in proportional reduction of heterozygosity, so it is un-exploitable in pure line. The epistatic variance is also reduce on selfing but its additive $\mathrm{x}$ additive remain constant, which is fixable.

The estimate of $\sigma^{2} \mathrm{~g}$ and $\sigma^{2} \mathrm{~s}$ and their ratio $\sigma^{2}$ $\mathrm{g} / \sigma^{2} \mathrm{~s}$ indicated a predominant role of additive gene action and non-additive gene action in $\mathrm{F}_{1}$ and $\mathrm{F}_{2}$ generation respectively. The different estimate obtained $I_{1}$ and $F_{2}$ generation grow in the same environment may be attribute to the restricted sampling in the total variability available in $F_{2}$ or may be due to linkage. Robinson et al., (1960) reported that if there 
was preponderance of repulsion phase of linkage, additive genetic variance could increase (i.e. non-additive to additive) as the generation were advance and if the linkage phase was predominantly coupling, additive genetic variance could decrease (i.e. additive to non-additive). The estimated value of $\sigma 2 \mathrm{~g}$ were higher than those of $\sigma 2 \mathrm{~g}, \sigma 2 \mathrm{r}$ indicating the predominance of additive gene action for days to $50 \%$ flowering, in F1 generation; plant height in F2 generation. Which indicated the predominance of additive gene action for these characters. Singh et.al. (2004). The value of $\sigma 2$ sca and $\sigma 2$ rca were higher than those of $\sigma 2 \mathrm{~g}$, indicating the predominance of non-additive gene action for number of primary branch, capsule size, day to maturity, number of seed per capsule, 1000 seed weight, oil content, all fatty acids in both generation; seed yield per plant in $F_{2}$ generation. The ratio $\sigma 2 \mathrm{~g} / \sigma 2 \mathrm{~s}$ was observed more than unity or closer to unity for days to $50 \%$ flowering in $\mathrm{F}_{1}$ and plant height and number of primary branch in $F_{2}$ generation which showed preponderance of additive gene action while rest traits showed preponderance of non-additive gene action.

\section{Combining ability}

\section{General combining ability}

The information regarding gca effect of parents is of prime importance as is help in successful prediction of genetic potentiality of crosses which produce desirable individuals in segregating generation as the choice of parents for hybridization is normally based on per se performance. The gca effect of parents was identified as good general combiner for all the characters in both generation. Parent KL-213 was found good general combiner for characters stearic acid, oleic acid and linoleic acid; OLC-60 was found good general combiner for characters plant height, days to $50 \%$ flowering, oil content, palmitic acid and stearic acid; Padmini was found good general combiner for characters plant height, days to $50 \%$ flowering, number of capsule per plant, capsule size, days to maturity, 1000 seed weight, seed yield per plant, oil content and oleic acid; RKY-19 was found good general combiner for characters plant height, days to $50 \%$ flowering, leaf area, days to maturity and linoleic acid; S-36 was found good general combiner for characters stearic acid and linoleic acid; SJKO-50 was found good general combiner for characters days to maturity and 1000 seed weight; TL-11 was found good general combiner for number of capsules per plant and linolenic acid; TL-27 was found good general combiner for leaf area, oil content and linolenic acid.

It indicated that per se performance of parents would provide an indication of their general combining ability for the utilization of them in hybridization programme.

The analysis of variance table for Method 1, Model I (parents and one set of $\mathrm{F}_{1} \mathrm{~s}$ and its reciprocal) with expectations of mean sum of square is as follows

\begin{tabular}{|l|l|l|l|l|l|}
\hline Source & d f & S.S. & M.S.S. & Expectations of M.S.S. & 'F' test \\
\hline Gca & $(n-1)$ & $S_{g}$ & $M_{g}$ & $\sigma^{2} e+2 n /(n-1) \sigma_{g}^{2}$ & $\begin{array}{l}M_{g} / M_{e} \text { for } n-1, \quad(b- \\
1)(c-1)(r-1) d f\end{array}$ \\
\hline Sca & $n(n-1) / 2$ & $S_{s}$ & $M_{s}$ & $\left.\sigma^{2} e+2 / n(n-1)\right) \sigma^{2} s_{i j}$ & $\begin{array}{l}M_{s} / M_{e} \text { for } n(n-1) / 2, \\
(b-1)(c-1)(r-1) d f\end{array}$ \\
\hline reciprocals & $n(n-1) / 2$ & $S_{r}$ & $M_{r}$ & $\left.\sigma^{2} e+2 / n(n-1)\right) \sigma^{2} r_{j i}$ & $\begin{array}{l}M_{r} / M_{e} \text { for } n(n-1) / 2, \\
(b-1)(c-1)(r-1) d f\end{array}$ \\
\hline Error & $(b-1)(c-1)(r-1)$ & $S_{e}$ & $M_{e}^{\prime}$ & $\sigma^{2}$ & \\
\hline
\end{tabular}


Table.1 (a) Analysis of variance for combining ability in 8 parent diallel cross (parents and their $\mathrm{F}_{1} \mathrm{~s}$ ) among $16^{\text {th }}$ characters in Linseed

\begin{tabular}{|c|c|c|c|c|c|c|c|c|c|}
\hline $\begin{array}{l}\text { Source of } \\
\text { variation }\end{array}$ & d.f. & $\begin{array}{c}\text { Plant } \\
\text { height } \\
(\mathrm{cm})\end{array}$ & $\begin{array}{c}\text { Day } \\
\text { to50\% flo } \\
\text { wering }\end{array}$ & leaf area & $\begin{array}{l}\text { No. of } \\
\text { primary } \\
\text { branch }\end{array}$ & $\begin{array}{c}\text { No.of } \\
\text { capsules } \\
\text { per plant }\end{array}$ & $\begin{array}{c}\text { Capsule } \\
\operatorname{size}(\mathrm{mm})\end{array}$ & $\begin{array}{c}\text { Days to } \\
\text { maturit } \\
y\end{array}$ & $\begin{array}{c}\text { No. of } \\
\text { seed per } \\
\text { capsule }\end{array}$ \\
\hline GCA & 7 & $291.90 * *$ & $151.17^{* *}$ & $0.08^{* *}$ & $0.63^{* *}$ & $494.68 * *$ & $0.34 * *$ & $39.97 * *$ & $1.23^{* *}$ \\
\hline SCA & 28 & $19.34 * *$ & $5.41 * *$ & $0.02 * *$ & $0.34 * *$ & $103.06 * *$ & $0.06^{* *}$ & $14.34 * *$ & $0.55^{* *}$ \\
\hline reciprocal & 28 & $12.01 * *$ & $11.83^{* *}$ & $0.55^{*}$ & $0.32 * *$ & $7.74 * *$ & $0.09 * *$ & $6.71^{* *}$ & $0.60^{* *}$ \\
\hline Error & 126 & 0.75 & 0.96 & 0.00 & 0.08 & 2.86 & 0.03 & 1.52 & 0.14 \\
\hline$\sigma^{2} g$ & & 18.19 & 9.38 & 0.00 & 0.03 & 30.73 & 0.01 & 2.40 & 0.06 \\
\hline$\sigma^{2} s$ & & 18.58 & 4.45 & 0.01 & 0.25 & 100.19 & 0.03 & 12.82 & 0.41 \\
\hline $\boldsymbol{\sigma}^{2}$ reciprocal & & 5.63 & 5.43 & 0.00 & 0.12 & 2.44 & 0.02 & 2.59 & 0.23 \\
\hline$\left(\sigma^{2} g / \sigma^{2} s\right)$ & & 0.97 & 2.10 & 0.25 & 0.13 & 0.30 & 0.53 & 0.18 & 0.16 \\
\hline
\end{tabular}

\begin{tabular}{|c|c|c|c|c|c|c|c|c|c|}
\hline $\begin{array}{l}\text { Source of } \\
\text { variation }\end{array}$ & d..f. & $\begin{array}{l}1000 \text { seed } \\
\text { weight }\end{array}$ & $\begin{array}{l}\text { Seed yield } \\
\text { per plant }\end{array}$ & $\begin{array}{c}\text { Oil } \\
\text { content } \\
\%\end{array}$ & $\begin{array}{c}\text { Palmitic } \\
\text { acid }\end{array}$ & $\begin{array}{c}\text { Stearic } \\
\text { acid }\end{array}$ & $\begin{array}{l}\text { Oleic } \\
\text { acid }\end{array}$ & $\begin{array}{c}\text { Linoleic } \\
\text { acid }\end{array}$ & $\begin{array}{l}\text { Lenolenic } \\
\text { acid }\end{array}$ \\
\hline GCA & 7 & $2.45 * *$ & $4.52 * *$ & $19.67 * *$ & $6.20 * *$ & $15.91 * *$ & $29.94 * *$ & $31.72 * *$ & $102.83^{* *}$ \\
\hline SCA & 28 & $1.09 * *$ & $1.18^{* * *}$ & $4.74 * *$ & $15.49 * *$ & $19.58 * *$ & $9.19 * *$ & $22.20 * *$ & $40.23 * *$ \\
\hline reciprocal & 28 & $0.39 * *$ & $0.59 * *$ & $3.06 * *$ & $13.36 * *$ & $9.34 * *$ & $22.82 * *$ & $12.03 * *$ & $22.41 * *$ \\
\hline Error & 126 & 0.03 & 0.01 & 0.30 & 0.40 & 0.35 & 0.41 & 0.31 & 0.47 \\
\hline$\sigma^{2} g$ & & 0.15 & 0.28 & 1.21 & 0.36 & 0.97 & 1.84 & 1.96 & 6.39 \\
\hline$\sigma^{2} s$ & & 1.06 & 1.17 & 4.43 & 15.09 & 19.23 & 8.78 & 21.88 & 39.76 \\
\hline$\sigma^{2}$ reciprocal & & 0.18 & .028 & 1.37 & 6.47 & 4.49 & 11.20 & 5.86 & 10.97 \\
\hline$\left(\sigma^{2} g / \sigma^{2} s\right)$ & & 0.14 & 0.24 & 0.27 & 0.02 & 0.05 & 0.21 & 0.08 & 0.16 \\
\hline
\end{tabular}

Note: * significant at $\mathrm{p}=0.05$ and $* *$ significant at $\mathrm{p}=0.01$ 
Table.1 (b) Analysis of variance for combining ability in 8 parent diallel cross (parents and their $\mathrm{F}_{2} \mathrm{~s}$ ) among $16^{\text {th }}$ characters in Linseed

\begin{tabular}{|l|l|l|l|l|l|l|l|l|l|}
\hline $\begin{array}{l}\text { Source of } \\
\text { variation }\end{array}$ & $\boldsymbol{d} . . f$. & $\begin{array}{c}\text { Plant } \\
\text { height } \\
\mathbf{c m})\end{array}$ & $\begin{array}{c}\text { Day } \\
\text { to50\%flo } \\
\text { wering }\end{array}$ & leaf area & $\begin{array}{c}\text { No. of } \\
\text { primary } \\
\text { branch }\end{array}$ & $\begin{array}{c}\text { No.of } \\
\text { capsules } \\
\text { per plant }\end{array}$ & $\begin{array}{c}\text { Capsule } \\
\text { size(mm) }\end{array}$ & $\begin{array}{c}\text { Days to } \\
\text { maturit } \\
\mathbf{y}\end{array}$ & $\begin{array}{c}\text { No. of } \\
\text { seed / } \\
\text { capsule }\end{array}$ \\
\hline GCA & 7 & $231.46^{* *}$ & $44.62^{* *}$ & $0.04^{* *}$ & $0.37^{*}$ & $382.89^{* *}$ & $0.40^{* *}$ & $77.48^{* *}$ & $0.57^{*}$ \\
\hline SCA & 28 & $9.24^{* *}$ & $6.20^{* *}$ & $0.03^{* *}$ & 0.15 & $38.49^{* *}$ & $0.18^{* *}$ & $8.23^{* *}$ & $1.08^{* *}$ \\
\hline reciprocal & 28 & $14.35^{* *}$ & $6.77^{* *}$ & $0.06^{* *}$ & $0.34^{* *}$ & $23.55^{* *}$ & $0.12^{* *}$ & $10.89^{* *}$ & $1.16^{* *}$ \\
\hline Error & 126 & 0.58 & 0.61 & 0.00 & 0.14 & 3.28 & 0.03 & 0.88 & 0.22 \\
\hline $\boldsymbol{\sigma}^{\mathbf{2}} \mathbf{g}$ & & 14.43 & 2.75 & 0.00 & 0.01 & 23.72 & 0.02 & 4.78 & 0.02 \\
\hline $\boldsymbol{\sigma}^{\mathbf{2}} \mathbf{s}$ & & 8.66 & 5.59 & 0.02 & 0.01 & 35.21 & 0.14 & 7.35 & 0.86 \\
\hline $\boldsymbol{\sigma}^{\mathbf{2}}$ reciprocal & & 6.88 & 3.07 & 0.02 & 0.09 & 10.13 & 0.04 & 5.00 & 0.47 \\
\hline $\left.\mathbf{\sigma}^{\mathbf{2}} \mathbf{g} / \boldsymbol{\sigma}^{\mathbf{2}} \mathbf{s}\right)$ & & 1.66 & 0.49 & 0.10 & 1.11 & 0.67 & 0.15 & 0.65 & 0.02 \\
\hline
\end{tabular}

\begin{tabular}{|c|c|c|c|c|c|c|c|c|c|}
\hline $\begin{array}{l}\text { Source of } \\
\text { variation }\end{array}$ & d..f. & $\begin{array}{l}1000 \text { seed } \\
\text { weight }\end{array}$ & $\begin{array}{l}\text { Seed yield } \\
\text { per plant }\end{array}$ & $\begin{array}{c}\text { Oil } \\
\text { content } \\
\%\end{array}$ & $\begin{array}{c}\text { Palmitic } \\
\text { acid }\end{array}$ & $\begin{array}{c}\text { Stearic } \\
\text { acid }\end{array}$ & $\begin{array}{l}\text { Oleic } \\
\text { acid }\end{array}$ & $\begin{array}{c}\text { Linoleic } \\
\text { acid }\end{array}$ & $\begin{array}{l}\text { Lenolenic } \\
\text { acid }\end{array}$ \\
\hline GCA & 7 & $2.04 * *$ & $2.84 * *$ & $29.38 * *$ & $12.41 * *$ & $13.39 * *$ & $17.29 * *$ & $31.62 * *$ & $120.26^{* *}$ \\
\hline SCA & 28 & $0.49 * *$ & $0.57 * *$ & $8.77 * *$ & $14.10 * *$ & $6.57 * *$ & $12.27 * *$ & $16.25 * *$ & $38.04 * *$ \\
\hline reciprocal & 28 & $0.77 * *$ & $0.87 * *$ & $8.63 * *$ & $12.17 * *$ & $11.76^{* *}$ & $10.59 * *$ & $7.99 * *$ & $50.14 * *$ \\
\hline Error & 126 & 0.01 & 0.01 & 0.24 & 0.37 & 0.38 & 0.36 & 0.38 & 0.53 \\
\hline$\sigma^{2} g$ & & 0.12 & 0.17 & 1.82 & 0.75 & 0.81 & 1.05 & 1.95 & 7.48 \\
\hline$\sigma^{2} s$ & & 0.48 & 0.55 & 8.52 & 13.73 & 6.19 & 11.90 & 15.86 & 37.51 \\
\hline$\sigma^{2}$ reciprocal & & 0.37 & 0.43 & 4.19 & 5.90 & 5.69 & 5.11 & 3.80 & 24.80 \\
\hline$\left(\sigma^{2} g / \sigma^{2} s\right)$ & & 0.26 & 0.31 & 0.21 & 0.05 & 0.13 & 0.08 & 0.12 & 0.19 \\
\hline
\end{tabular}

Note: * significant at $\mathrm{p}=0.05$ and $* *$ significant at $\mathrm{p}=0.01$ 
Table.2 Estimates of mean performance and gca effect of 8 diallel parents for $16^{\text {th }}$ characters in Linseed

\begin{tabular}{|c|c|c|c|c|c|c|c|c|c|c|c|c|}
\hline \multirow[t]{3}{*}{ Parents } & \multicolumn{3}{|c|}{ Plant height (cm) } & \multicolumn{3}{|c|}{ Day to50\% flowering } & \multicolumn{3}{|c|}{ leaf area } & \multicolumn{3}{|c|}{ Number of primary branch } \\
\hline & \multicolumn{2}{|c|}{ GCA effect } & \multirow{2}{*}{ Mean } & \multicolumn{2}{|c|}{ GCA effect } & \multirow{2}{*}{ Mean } & \multicolumn{2}{|c|}{ GCA effect } & \multirow{2}{*}{$\begin{array}{l}\text { Mea } \\
\text { n }\end{array}$} & \multicolumn{2}{|c|}{ GCA effect } & \multirow{2}{*}{$\begin{array}{l}\text { Mea } \\
\text { n }\end{array}$} \\
\hline & $\mathbf{F}_{1}$ & $\mathbf{F}_{2}$ & & $\mathbf{F}_{1}$ & $\mathbf{F}_{2}$ & & $\mathbf{F}_{1}$ & $\mathbf{F}_{2}$ & & $\mathbf{F}_{1}$ & $\mathbf{F}_{2}$ & \\
\hline KL-213 & $3.12 * *$ & $3.93 * *$ & 86.53 & $3.92 * *$ & $2.38 * *$ & 82.33 & $-0.07 * *$ & -0.02 & 1.04 & $0.28 * *$ & 0.10 & 5.33 \\
\hline OLC-60 & $-1.79 * *$ & $-1.33^{* *}$ & 73.76 & $-2.51 * *$ & $-1.12^{* *}$ & 68.33 & $-0.07 * *$ & -0.03 & 1.21 & -0.06 & 0.04 & 5.33 \\
\hline PADMINI & $-9.10^{* * *}$ & $-7.25^{* *}$ & 59.43 & $-4.49 * *$ & $-2.14 * *$ & 67.33 & $-0.05^{*}$ & $-0.06^{* *}$ & 1.27 & $-0.17 *$ & $-0.20 *$ & 5.33 \\
\hline RKY-19 & $-2.30^{* *}$ & $-3.49 * *$ & 68.66 & $-3.41 * *$ & $-2.20 * *$ & 66.66 & $0.05^{*}$ & $0.09 * *$ & 1.55 & -0.00 & 0.00 & 5.00 \\
\hline S-36 & 1.74 & $1.92 * *$ & 79.90 & $2.60^{* *}$ & $0.60 * *$ & 78.00 & $0.04 *$ & 0.01 & 1.49 & $0.18 * *$ & 0.06 & 5.33 \\
\hline SJKO-50 & $3.21 * *$ & $0.88^{* *}$ & 80.76 & $0.71 * *$ & $1.29 * *$ & 75.66 & $-0.06 * *$ & -0.03 & 1.26 & -0.00 & 0.00 & 5.33 \\
\hline TL-11 & $2.80 * *$ & $3.17 * *$ & 81.36 & $1.92 * *$ & $1.04 * *$ & 77.66 & $0.09 * *$ & 0.00 & 1.61 & 0.12 & $0.21 *$ & 6.33 \\
\hline TL-27 & $2.30 * *$ & $2.16^{* *}$ & 82.36 & $1.25 * *$ & 0.13 & 76.33 & $0.07 * *$ & $0.05 * *$ & 1.71 & $-0.33^{* *}$ & $-0.24 * *$ & 5.00 \\
\hline $\mathrm{SE} \pm(\mathbf{G i})$ & 0.48 & 0.42 & & 0.54 & 0.43 & & 0.05 & 0.04 & & 0.16 & 0.21 & \\
\hline $\mathbf{S E} \pm(\mathbf{G i}-\mathbf{G j})$ & 0.72 & 0.63 & & 0.82 & 0.65 & & 0.07 & 0.07 & & 0.24 & 0.32 & \\
\hline
\end{tabular}

Table.2 Continued

\begin{tabular}{|c|c|c|c|c|c|c|c|c|c|c|c|c|}
\hline \multirow[t]{3}{*}{ Parents } & \multicolumn{3}{|c|}{$\begin{array}{c}\text { Number of capsules per } \\
\text { plant }\end{array}$} & \multicolumn{3}{|c|}{ Capsule size $(\mathrm{mm})$} & \multicolumn{3}{|c|}{ Days to maturity } & \multicolumn{3}{|c|}{ No. of seed per capsule } \\
\hline & \multicolumn{2}{|c|}{ GCA effect } & \multirow[t]{2}{*}{ Mean } & \multicolumn{2}{|c|}{ GCA effect } & \multirow[t]{2}{*}{ Mean } & \multicolumn{2}{|c|}{ GCA effect } & \multirow[t]{2}{*}{ Mean } & \multicolumn{2}{|c|}{ GCA effect } & \multirow{2}{*}{$\begin{array}{l}\text { Mea } \\
\text { n }\end{array}$} \\
\hline & $\mathbf{F}_{1}$ & $\mathbf{F}_{2}$ & & $\mathbf{F}_{1}$ & $\mathbf{F}_{2}$ & & $\mathbf{F}_{1}$ & $\mathbf{F}_{2}$ & & $\mathbf{F}_{1}$ & $\mathbf{F}_{2}$ & \\
\hline KL-213 & $-4.47 * *$ & $-4.87 * *$ & 62.66 & $-0.18 * *$ & $-0.23 * *$ & 6.00 & $1.28 * *$ & $2.67 * *$ & 144.33 & -0.06 & 0.19 & 8.00 \\
\hline OLC-60 & $-0.82 *$ & $1.00 *$ & 74.33 & $-0.19 * *$ & $-0.09 *$ & 6.16 & 0.09 & $-1.43 * *$ & 133.0 & 0.00 & 0.06 & 8.33 \\
\hline $\begin{array}{l}\text { PADMIN } \\
\text { I }\end{array}$ & $11.48 * *$ & $10.54 * *$ & 86.33 & $0.19 * *$ & $0.29 * *$ & 6.83 & $-1.65 * *$ & $-1.41 * *$ & 128.33 & $0.39 * *$ & 0.21 & 8.00 \\
\hline RKY-19 & $1.67 * *$ & $-1.35 * *$ & 70.66 & -0.06 & $-0.11 *$ & 6.26 & $-2.23 * *$ & $-2.26 * *$ & 131.66 & $0.28 * *$ & $-0.28 *$ & 9.00 \\
\hline S-36 & $-4.82 * *$ & $-1.91 * *$ & 71.66 & 0.00 & $0.09 *$ & 6.83 & -0.38 & $-0.80 * *$ & 132.33 & $-.38 * *$ & -0.16 & 8.00 \\
\hline SJKO-50 & $-353 * *$ & $-4.02 * *$ & 66.66 & $0.19 * *$ & 0.03 & 7.00 & $-0.88 * *$ & $-1.89 * *$ & 131.66 & 0.09 & 0.00 & 7.33 \\
\hline TL-11 & $3.79 * *$ & $2.52 * *$ & 80.00 & 0.02 & 0.00 & 7.06 & $1.64 * *$ & $2.85 * *$ & 139.00 & 0.05 & 0.15 & 8.00 \\
\hline TL-27 & $-3.28 * *$ & $-1.89 * *$ & 63.66 & 0.03 & 0.03 & 6.53 & $2.12^{* *}$ & $2.27 * *$ & 138.00 & $-38 * *$ & -0.18 & 7.33 \\
\hline $\mathrm{SE} \pm(\mathbf{G i})$ & 0.93 & 1.00 & & 0.10 & 0.10 & & 1.03 & 0.51 & & 0.20 & 0.26 & \\
\hline $\begin{array}{l}\text { SE } \pm(G i- \\
\text { Gj) }\end{array}$ & 1.41 & 1.51 & & 0.15 & 0.15 & & 0.68 & 0.78 & & 0.31 & 0.39 & \\
\hline
\end{tabular}


Table.2 Continued

\begin{tabular}{|c|c|c|c|c|c|c|c|c|c|c|c|c|}
\hline \multirow[t]{3}{*}{ Parents } & \multicolumn{3}{|c|}{1000 seed weight } & \multicolumn{3}{|c|}{ Seed yield per plant } & \multicolumn{3}{|c|}{ Oil content \% } & \multicolumn{3}{|c|}{ Palmitic acid } \\
\hline & \multicolumn{2}{|c|}{ GCA effect } & \multirow[t]{2}{*}{ Mean } & \multicolumn{2}{|c|}{ GCA effect } & \multirow[t]{2}{*}{ Mean } & \multicolumn{2}{|c|}{ GCA effect } & \multirow[t]{2}{*}{ Mean } & \multicolumn{2}{|c|}{ GCA effect } & \multirow{2}{*}{$\begin{array}{l}\text { Mea } \\
\text { n }\end{array}$} \\
\hline & $\mathbf{F}_{1}$ & $\mathbf{F}_{2}$ & & $\mathbf{F}_{1}$ & $\mathbf{F}_{2}$ & & $\mathbf{F}_{1}$ & $\mathbf{F}_{2}$ & & $\mathbf{F}_{1}$ & $\mathbf{F}_{2}$ & \\
\hline KL-213 & $-0.53 * *$ & $-0.34 * *$ & 4.51 & $-0.52 * *$ & $-0.38 * *$ & 3.15 & $-1.06^{* *}$ & $-1.24 * *$ & 33.54 & $-0.62 * *$ & $-0.38 * *$ & 6.72 \\
\hline OLC-60 & -0.06 & $-0.17 *$ & 5.72 & -0.00 & $0.15 * *$ & 4.56 & $0.48 * *$ & $0.39 * *$ & 39.26 & $1.26 * *$ & $0.43 * *$ & 13.19 \\
\hline $\begin{array}{l}\text { PADMIN } \\
\text { I }\end{array}$ & $0.52 * *$ & $0.45 * *$ & 7.40 & $1.52 * *$ & $0.88 * *$ & 5.30 & $1.93 * *$ & $2.69 * *$ & 42.52 & $-0.57 * *$ & 0.23 & $8 / .55$ \\
\hline RKY-19 & $-0.16^{* *}$ & $-0.50 * *$ & 5.05 & $0.11 * *$ & $-0.29 * *$ & 3.65 & $1.59 * *$ & $-0.69 * *$ & 36.38 & $-0.36^{*}$ & $0.43 * *$ & 8.92 \\
\hline S-36 & -0.01 & $-0.07 *$ & 6.82 & $-0.46 * *$ & $-0.08 * *$ & 4.44 & $-0.27 *$ & $-1.44 * *$ & 34.65 & $0.34 *$ & -0.06 & 11.46 \\
\hline SJKO-50 & $0.55^{*}$ & $0.51 * *$ & 7.89 & $0.12 * *$ & 0.04 & 3.75 & $0.50 * *$ & 0.02 & 36.67 & 0.00 & -0.08 & 6.86 \\
\hline TL-11 & -0.41 & $0.14 * *$ & 5.84 & -0.02 & $0.09 * *$ & 4.13 & $-0.55^{* *}$ & $-0.67 * *$ & 38.68 & 0.25 & $1.23 * *$ & 19.00 \\
\hline TL-27 & 0.11 & $-0.12 *$ & 7.00 & $-0.36 * *$ & $-0.14 * *$ & 3.66 & $0.56 * *$ & $0.94 * *$ & 40.28 & $-0.33^{*}$ & $-1.81 * *$ & 6.38 \\
\hline $\mathrm{SE} \pm(\mathrm{Gi})$ & 0.10 & 0.06 & & 0.06 & 0.07 & & 0.30 & 0.27 & & 0.35 & 0.33 & \\
\hline $\begin{array}{l}\text { SE } \pm(G i- \\
\text { Gj) }\end{array}$ & 0.15 & 0.10 & & 0.09 & 0.11 & & 0.46 & 0.41 & & 0.53 & 0.51 & \\
\hline
\end{tabular}

Table.2 Continued

\begin{tabular}{|c|c|c|c|c|c|c|c|c|c|c|c|c|}
\hline \multirow[t]{3}{*}{ Parents } & \multicolumn{3}{|c|}{ Stearic acid } & \multicolumn{3}{|c|}{ Oleic acid } & \multicolumn{3}{|c|}{ Linoleic acid } & \multicolumn{3}{|c|}{ Linolenic acid } \\
\hline & \multicolumn{2}{|c|}{ GCA effect } & \multirow[t]{2}{*}{ Mean } & \multicolumn{2}{|c|}{ GCA effect } & \multirow[t]{2}{*}{ Mean } & \multicolumn{2}{|c|}{ GCA effect } & \multirow{2}{*}{$\begin{array}{l}\text { Mea } \\
\text { n }\end{array}$} & \multicolumn{2}{|c|}{ GCA effect } & \multirow{2}{*}{$\begin{array}{l}\text { Mea } \\
\text { n }\end{array}$} \\
\hline & $\mathbf{F}_{1}$ & $\mathbf{F}_{2}$ & & $\mathbf{F}_{1}$ & $\mathbf{F}_{2}$ & & $\mathbf{F}_{1}$ & $\mathbf{F}_{2}$ & & $\mathbf{F}_{1}$ & $\mathbf{F}_{2}$ & \\
\hline KL-213 & $0.58 * *$ & $0.29 *$ & 5.85 & $0.99 * *$ & $0.69 * *$ & 20.38 & $0.77 * *$ & $1.11 * *$ & 14.54 & $-1.48 * *$ & $-1.74 * *$ & 52.49 \\
\hline OLC-60 & $1.33 * *$ & $0.53 * *$ & 13.35 & $-0.80 * *$ & -0.01 & 14.51 & $0.99 * *$ & $-0.46 * *$ & 9.46 & $-2.46 * *$ & $-0.41 *$ & 49.50 \\
\hline PADMINI & $-0.44 * *$ & $-0.45 * *$ & 7.62 & $1.42 * *$ & $1.22 * *$ & 20.43 & $-1.44 * *$ & $-0.93 * *$ & 4.73 & -0.06 & -0.06 & 58.66 \\
\hline RKY-19 & $-0.98 * *$ & 0.19 & 8.27 & 0.04 & $0.42 * *$ & 12.66 & $1.00 * *$ & $1.08 * *$ & 21.58 & $0.48 * *$ & $-2.15 * *$ & 48.55 \\
\hline S-36 & $1.23^{* *}$ & $0.87 * *$ & 10.75 & $-1.09 * *$ & $-0.53 * *$ & 10.60 & $2.16^{* *}$ & $0.40 * *$ & 12.54 & $-2.24 * *$ & $-0.77 * *$ & 54.63 \\
\hline SJKO-50 & $-0.38 * *$ & $0.95 * *$ & 5.95 & $1.92 * *$ & -0.01 & 19.28 & $-0.68 * *$ & $2.11 * *$ & 17.30 & $-1.71 * *$ & $-2.98 * *$ & 50.60 \\
\hline TL-11 & 0.08 & $-0.61 * *$ & 10.20 & $-2.08 * *$ & $-2.21 * *$ & 8.73 & $-1.29 * *$ & $-1.39 * *$ & 13.42 & $3.25 * *$ & $3.00 * *$ & 48.63 \\
\hline TL-27 & $-1.41 * *$ & $-1.78 * *$ & 5.41 & $-0.41 * *$ & $0.43 * *$ & 17.43 & $-1.53 * *$ & $-1.92 * *$ & 13.45 & $4.23 * *$ & $5.12 * *$ & 57.31 \\
\hline $\mathrm{SE} \pm(\mathbf{G i})$ & 0.33 & 0.34 & & 0.35 & 0.33 & & 0.31 & 0.34 & & 0.38 & 0.40 & \\
\hline $\mathrm{SE} \pm(\mathbf{G i}-\mathbf{G j})$ & 0.49 & 0.51 & & 0.54 & 0.50 & & 0.47 & 0.52 & & 0.57 & 0.61 & \\
\hline
\end{tabular}

Note: $*$ significant at $\mathrm{p}=0.05$ and $* *$ significant at $\mathrm{p}=0.01$ 
Int.J.Curr.Microbiol.App.Sci (2017) 6(11): 1504-1516

Table.3 Heterosis range, number of desirable hybrids and best hybrids (better parental) for 16 traits in linseed

\begin{tabular}{|c|c|c|c|c|}
\hline Traits & Heterosis type & Heterosis range & Number of desired hybrids & Best hybrids \\
\hline Plant height $(\mathrm{cm})$ & $\mathrm{BP}$ & -30.08 to 8.30 & 48 & KL-213 x Padmini \\
\hline Days to $50 \%$ flowering & $\mathrm{BP}$ & -21.00 to 1.17 & 35 & S-36 x TL-11 \\
\hline Leaf area & $\mathrm{BP}$ & -26.60 to 43.85 & 6 & KL-213 x OLC-60 \\
\hline $\begin{array}{l}\text { Number of primary } \\
\text { branches per plant }\end{array}$ & $\mathrm{BP}$ & -21.05 to 40.00 & 13 & TL-27 x RKY-19 \\
\hline Capsule size & $\mathrm{BP}$ & -14.15 to 9.19 & 1 & OLC-60 x KL-213 \\
\hline Days to maturity & $\mathrm{BP}$ & -12.24 to 2.50 & 34 & TL-11 x KL-213 \\
\hline Seed yield per plant (g) & $\mathrm{BP}$ & -27.78 to 71.73 & 26 & RKY-19 x SJKO-50 \\
\hline Oil content $(\%)$ & $\mathrm{BP}$ & -17.17 to 13.28 & 5 & SJKO-50 x OLC-60 \\
\hline Palmitic acid & $\mathrm{BP}$ & -81.86 to 125.36 & 13 & TL-27 x SJKO-50 \\
\hline Stearic acid & $\mathrm{BP}$ & -78.80 to 107.34 & 10 & KL-213 x TL-27 \\
\hline Oleic acid & $\mathrm{BP}$ & -60.44 to 54.17 & 7 & RKY-19 x S-36 \\
\hline Linoleic acid & $\mathrm{BP}$ & -90.90 to 76.48 & 9 & Padmini x OLC-60 \\
\hline Lenolenic acid & $\mathrm{BP}$ & -28.24 to 36.72 & 17 & RKY-19 x TL-11 \\
\hline
\end{tabular}


Combining ability describes the breeding value of parental lines to produce hybrids (Romanus et al., 2008). The general combining ability has been equated with additive gene action and specific combining ability with non-additive gene action (Griffing 1956 a). The analysis of variance for combining ability was done for all the 16 characters (Table 2). Highly significant variances, of general, specific and reciprocal combining ability, were observed which indicated the importance of both additive and non-additive gene effects for all the in traits both generations.

\section{Specific combining ability effects}

In general, sca effects do not make any worthwhile contributions in the improvement of self-fertilizing crops expect where there is possibility of commercial exploitation of heterosis. Breeders' interest normally, however, rests in obtaining transgressive segregants through crosses in order to produce homozygous lines in autogamous crops like linseed. Jinks and Jones (1958) further emphasized that superior per se performance of the hybrids might indicate their ability to produce transgressive hybrids may not indicate their ability to between heterosis and non-segregants due to close correspondence between heterosis and nonadditive gene effects. Therefore, study of sca and rca in segregating generating generation would be a better preposition for heterosis breeding. Padmini $\times$ SJKO-50 for plant height; KL-213 $\times$ OLC-60, KL-213 $\times$ RKY19, S-36 $\times$ SJKO-50 for leaf area; KL-213 $\times$ SJKO-50, KL-213 $\times$ S-36, S-36 $\times$ TL-11 number of primary branch per plant; RKY-19 $\times$ TL-11, Padmini $\times$ TL-27, KL-213× Padmini for number of capsules per plant; S$36 \times$ TL-27 for capsule size; Padmini $\times$ RKY19 for day to maturity; OLC-60 $\times$ SJKO-50, RKY-19 $\times$ TL-11 number of seed per sapsule; OLC-60 $\times$ SJKO-50, Padmini $\times$ RKY-19,
RKY-19 $\times$ SJKO-50, TL-11 $\times$ TL-27 and KL$213 \times$ S-36 for 1000 seed weight; Padmini $\times$ RKY-19, OLC-60 $\times$ SJKO-50, Padmini $\times$ TL27, KL-213 $\times$ Padmini for seed yield per plant; Padmini $\times$ S-36, KL-213 $\times$ Padmini for oil content; Padmini $\times$ TL-27, SJKO-50 $\times$ TL11, Padmini $\times$ S-36 for palmitic acid; OLC-60 $\times$ Padmini, OLC-60 $\times$ S-36, SJKO-50 $\times$ TL11 for stearic acid; Padmini $\times$ TL-11 for oleic acid; RKY-19 $\times$ S-36, KL-213 $\times$ S-36 for linoleic acid and RKY-19 $\times$ TL-27, RKY-19 $\times$ TL-11, Padmini $\times$ TL-11 for linolenic acid were found good specific combiner as well as per se performance in $\mathrm{F}_{1}$ population. Padmini $\times$ SJKO-50 for plant height; Padmini $\times$ TL11, Padmini $\times$ TL-27 for days to $50 \%$ flowering; SJKO-50 $\times$ TL-27 for leaf area; KL-213 $\times$ S-36 for number of primary branch per plant; OLC-60 $\times$ TL-11, KL-213 $\times$ Padmini, Padmini $\times$ TL-27 number of capsules per plant; S-36 $\times$ TL-27, Padmini $\times$ SJKO-50 for capsule size; S-36 $\times$ SJKO-50, RKY-19 $\times$ SJKO-50 for days to maturity; OLC-60 $\times$ TL-27, Padmini $\times$ S-36 number of seed per capsule; Padmini $\times$ S-36, OLC- $60 \times$ TL-27, RKY-19 $\times$ SJKO-50 for seed yield per plant; RKY-19 × S-36, SJKO-50 × TL-11, Padmini $\times$ TL-27 for oil content; Padmini $\times$ RKY-19, OLC-60 × TL-11, KL-213 × SJKO50 for palmitic acid; S-36 $\times$ SJKO-50, KL$213 \times$ SJKO-50 stearic acid; OLC-60 $\times$ S-36, Padmini $\times$ TL-27, OLC-60 $\times$ TL-11, RKY-19 $\times$ TL-27 for oleic acid; KL-213 $\times$ Padmini, OLC-60 $\times$ RKY-19, S-36 $\times$ SJKO-50 for linoleic acid and KL-213 $\times$ TL-27, S-36 $\times$ TL-27 linolenic acid were found good specific combiner as well as per se performance in $\mathrm{F}_{2}$ population.

None of the crosses showed significant rca effects for the characters with the expression of plant height. The significant rca effects for days to $50 \%$ flowering in S-36 $\times$ Padmini; leaf area in OLC-60 × KL-213; number of seeds per capsule in TL-27 $\times$ RKY-19, TL-11 $\times$ RKY-19; oil content Padminix KL-213; 
palmitic acid in TL-27 $\times$ Padmini; stearic acid in TL-11 $\times$ SJKO-50. Oleic acid in TL-11 $\times$ Padmini; linoleic acid in S-36 $\times$ KL-213; linolenic acid in TL-11 $\times$ Padmini, TL-27 $\times$ RKY-19 in F1 generation. Day to $50 \%$ flowering in TL-11× KL-213; number of primary branches per plant in S-36 × KL-213; number of capsules per plant in Padminix KL-213, TL-11 $\times$ RKY-19; number of seeds per capsule in S-36 $\times$ Padmini; 1000 seed weight in RKY-19 x OLC-60; seed yield per plant in TL-27 $\times$ OLC-60, SJKO-50 $\times$ RKY19; oil content in S-36 $\times \mathrm{RKY}-19$, TL-11 $\times$ SJKO-50; palmitic acid in TL-11 $\times$ OLC-60; oleic acid in TL-27 $\times$ Padmini, TL-11 $\times$ OLC60 ; linoleic acid in RKY-19 $\times$ OLC-60 in F2 generation. It is noteworthy that the crosses, showing consistently positive sca effects also exhibitted positive significant heterosis. Thus, the results of the present study indicated some relationship between sca effects and heterosis. It is therefore suggested that sca performance may be considered as a criterion for selecting the best crosses in linseed. It may also be worthwhile to attempt bi-parental mating in the segregating generation among selected crosses to permit superior recombinations.

All the important crosses involving parents with high $\times$ average, average $\times$ /average and average $\times /$ low general combiners, indicated that non-additive type of gene actions, which are unfixable in nature were involved in selected cross combinations. The study demonstrates that both additive (fixable) and non-additive (non-fixable) components of genetic variances were involved in governing the inheritance of almost all the quantitative and quality traits, although additive genetic variance was predominant. Therefore, biparental mating and diallel selective mating which may allow intermating of the selects in different cycles and exploit both additive and non-additive gene effect could be useful in the genetic improvement of the characters of linseed. Inclusion of $F_{1}$ hybrids showing high sca and having parents with good gca, into multiple crosses, could also be a significant approach for tangible improvement of almost traits.

\section{Heterosis}

Heterosis breeding plays an important role in crop improvement for obtaining higher production. The degree of heterosis should preferably be measured by superior in $F_{1}$ hybrid over batter parent or best commercial variety. In the present investigation, heterosis was over batter parents for all the sixteen characters studied were found with all crosses. A wide variation of heterosis range, number of desired hybrids and best hybrid was found for most of the traits (Table 3). Singh et al., (2004) stated that the superiority of hybrids particularly over high parent is more useful for commercial exploitation of heterosis and also indicated the parental combinations capable of producing the highest level of transgressive segregants.

\section{References}

Dillman, AD., 1953. Classification of flax varieties, 1946 US Dept. of Agriculture, 1953.Series Information. Technical Bulletin/UNnited States Department of Agriculture.no. 1064. US Dept of Agriculture, Washington.

Griffing B. 1956 a. Concepts of specific and general combining ability in relation to diallel crossing systems. Australian Journal of Biological Sciences 9: 46393.

Jinks, J.L. and Jones, J.M. (1958). Estimation of components of heterosis. Genetics. 43: 223-234.

Romanus K G, Hussein S and Mashela W P. 2008. Combining ability analysis and association of yield and yield components among selected cowpea lines. Euphytica 162: 205-10. 
Singh H, Sharma S N and Sain R S. 2004. Heterosis studies for yield and its components in bread wheat over environments. Hereditas 141: 106-14.

Singh,-H-C; $\quad$ Dixit,-R-K; $\quad$ Pathak,-R-K; Rajendra-Singh; Nalini-Tiwari(2004) Genetic analysis of quality traits in linseed (Linum usitatissimum L.).

Viorica Mirela Popa, Alexandra Gruia Diana
Raba, Delia Dumbrava, Camelia Moldovan, Despina Bordean, Constantin Mateescu (2012) Fatty acids composition and oil characteristics of linseed (Linum usitatissimum L.) Banat's University of Agricultural Sciences and Veterinary Medicine, 300645 Timisoara, Calea Aradului, 119, Romania.

\section{How to cite this article:}

Shalendra Kumar, P.K. Singh, S.D. Dubey, S.K. Singh and Alankar Lamba. 2017. Heterosis and Combining Ability Analysis Oil Content Seed Yield and Its Component in Linseed. Int.J.Curr.Microbiol.App.Sci. 6(11): 1504-1516. doi: https://doi.org/10.20546/ijcmas.2017.611.178 